\title{
Cuidado, integralidade e atenção primária: articulação essencial para refletir sobre o setor saúde no Brasil
}

\author{
Care, integrality and primary care: essential articulation to reflect on
} the health sector in Brazil

Leonardo Carnut 1

RESUMO Este artigo descreve os elementos teórico-conceituais sobre cuidado, integralidade e Atenção Primária à Saúde, articulando-os e ressaltando a essencialidade destas ideias na estruturação do sistema de saúde no Brasil. Optou-se pelo formato ensaístico segundo os argumentos de Meneghetti e Adorno. Foram apresentados os conceitos de cuidado, integralidade e atenção primária, assim como algumas críticas de ordem teórico-prática. Foi demonstrada a existência de um fio condutor que os une, tornando-os um eixo estrutural do Sistema Único de Saúde, assim como os principais limites e possibilidades dessa articulação. Por fim, lançou-se uma brevíssima sugestão sobre o tema em tempos de crise econômico-política.

PALAVRAS-CHAVE Empatia. Atenção Primária à Saúde. Integralidade em saúde.

ABSTRACT This article describes the theoretical-conceptual elements about care, integrality and Primary Health Care, articulating them and emphasizing the essentiality of these ideas in the structuring of the health system in Brazil. It was chosen the essay format according to the arguments of Meneghetti and Adorno. The concepts of care, integrality and primary care were presented, as well as some critiques of theoretical-practical order. It has been demonstrated the existence of a guiding thread that unites them, making them a structural axis of the Unified Health System, as well as the main limits and possibilities of this articulation. Finally, a very brief suggestion was made on the subject in times of economic-political crisis.

KEYWORDS Empathy. Primary Health Care. Health integrality. 


\section{Introdução}

Pensar no cuidado como prática sanitária já é, em si, ter o outro como um todo. Os profissionais que escolhem a área da saúde, em algum momento, pensam o quanto de suas vidas precisarão dedicar a um 'outro', que não conhecerão previamente e que, portanto, será alvo do seu altruísmo, como prática profissional e de vida.

Hermeneuticamente falando, o 'cuidador' e o 'cuidado' não são símbolos de interações quaisquer, mas sim, de uma interação especial, na qual o 'afetar-se' consolida-se no 'querem bem' ao outro. Isto só é possível quando se entende o que é o 'bem' para o outro, e isto requer uma cosmovisão do universo do outro (BUB ET AL., 2006).

Ver o outro na sua cosmovisão é, em outras palavras, vê-lo como um ser integral. Na saúde, a palavra 'integral' (e sua substantivação 'integralidade') vem sendo a tônica da prestação dos serviços de saúde, que a advogam como objetivo a ser alcançado na produção de um novo cuidar. Mesmo sendo evocada em todos os níveis de atenção, a Atenção Primária à Saúde (APS) tem sido constantemente assinalada por diversos documentos oficiais (BRASIL, 1988; BRASIL, 1990; BRASIL, 2011) e pesquisas empíricas (SANTANA ET AL., 2010; SANTOS ET AL., 2007) como espaço privilegiado para o exercício e a coordenação da integralidade.

À guisa dessa discussão, este texto tem como objetivo descrever os elementos teórico-conceituais sobre cuidado, integralidade e APS, 'articulando-os' e ressaltando as evidências que caracterizam a essencialidade desta articulação, para um processo de produção em saúde que resgate o 'ser' humano.

Para tanto, optou-se pela modalidade textual de ensaio, devido à possibilidade de a orientação ser dada não pela busca das respostas e afirmações verdadeiras, mas pelas perguntas que possam orientar os sujeitos para as reflexões mais profundas (MENEGHETTI, 2011). Assim, como o objetivo deste ensaio é 'articular conceitos', o procedimento metodológico clássico parece não ser o mais adequado de se utilizar. Por isto, inspirou-se em Adorno (2003), quando este defende que o ensaio é um momento de escrita que favorece a ultrapassagem de fronteiras culturalmente demarcadas.

Assim, o texto foi dividido em oito seções, que, longe de esgotarem o tema, trazem consigo argumentos para refletir e ponderar sobre essa articulação e pensar em quais são os desafios postos na produção do trabalho em saúde, para que este se qualifique com vistas à melhoria da saúde das populações.

\section{Conceito de cuidado: o cuidado do outro e o cuidado de si}

Para o trabalho em saúde no âmbito da atenção primária, o profissional deve levar em consideração alguns conhecimentos necessários para o exercício de uma prática de saúde consoante aos princípios e diretrizes do Sistema Único de Saúde (SUS), assim como seu papel enquanto profissional de saúde. A categoria 'cuidado', mesmo alguns autores (VALE; PAGLUICA, 2011) defendendo-o como uma função precípua de uma categoria específica, neste ensaio, defende-se que é uma função que deve perpassar todas as categorias profissionais inseridas no âmbito da saúde. Logo, todas devem deter o 'cuidado' como objeto de sua práxis, levando em consideração que o entendimento da expressão 'deter saúde' depende de condições políticas, econômicas, ideológicas e tecnológicas, e, assim sendo, podem reconfigurar o que se entende por objeto da prática profissional de cada categoria. Então, em saúde, a tensão tradicionalmente se instalou na diferenciação entre tomar como objeto o 'cuidado' ou a 'intervenção' (CARNUT ET AL., 2016, P. 3). Intervir significa fazer algo para/em um 'outro' que desconsidera os elementos subjetivos da interação profissional-usuário. Em uma 
relação clínica que presa o outro como centro de sua prática, a categoria 'cuidado' é aquela que ressalta os elementos subjetivos dessa interação, valorizando-os na interpretação do processo saúde-adoecimento dos sujeitos. Assim, o cuidado emerge como categoria central na prática de quaisquer profissionais de saúde, exigindo, assim, que todos aqueles que se dedicam à área compreendam os elementos constitutivos do cuidado. Em Vale e Pagluica (2011), o cuidado é o zelo, a dedicação, a afeição, o preocupar-se com o outro. Neste sentido, o cuidado requer do profissional que deseja prestá-lo alguns atributos necessários, sendo eles: a ética nas relações humanas, a solidariedade e a confiança. Todo cuidado tem como objetivo o alívio, o conforto, podendo promover a cura, o bem- estar e, quiçá, a mudança de estilo de vida.

Em que pese a relevância do cuidado do profissional de saúde com o usuário, Bub et al. (2006) relataram que, tradicionalmente nas profissões da saúde, o cuidado (entendido como autocuidado) também está na centralidade da discussão, especialmente quando se constata a corresponsabilização do paciente no sucesso ou malogro da ação terapêutica. Assim, teorias sobre o autocuidado foram desenvolvidas com o intuito de definir quais os determinantes da cooperação do paciente, nas medidas a serem tomadas como autocuidado, para o sucesso da ação profissional. Mesmo sendo fundamental essa compreensão, as teorias sobre o autocuidado pareciam muito prescritivas, verticalizadas e normativas. Nelas, o autocuidado simbolizava aquilo que o profissional de saúde desejava que o paciente fizesse em sua ausência, e não necessariamente o que o paciente podia e queria fazer enquanto sujeito desejante. Assim, as discussões atuais sobre o autocuidado transmutaram-se para o que hoje se discute como o 'cuidado de si' (FOUCAULT, 2007), como forma de revisitar a subjetividade envolvida na relação clínica profissional-usuário, e como o sujeito que recebe o cuidado também deve ser produtor de bem-estar, em função dos desejos que o conduzem nas suas experiências de vida. Portanto, o cuidado de si trabalha a ideia de conversão, ou converter-se a partir do que é bom para si. Seria, assim, um 'pertencer a si mesmo', ou, em síntese, um aprender a ser 'seu'. Mesmo com essa complexa base epistemológica, o SUS já tinha, em sua concepção original, como ampliar o olhar para esse sujeito que deseja ter o protagonismo do seu cuidado como algo importante, o que ficou bastante evidente na concepção de integralidade descrita em seu arcabouço jurídico-institucional.

\section{Integralidade: polissemia e aplicabilidade}

A integralidade, no SUS, é uma concepção difusa e complexa, extremamente polissêmica (PINHEIRO; MATTOS, 2012), e que requer várias formas de operacionalização no cotidiano das práticas. A principal delas está inscrita na Constituição Federal de 1988, que relata o 'atendimento integral' (BRASIL, 1988) como a necessidade de compreender o indivíduo enquanto um todo holístico, um ser biopsicossocial em sua essência. Outro conceito de integralidade importante encontra-se na Lei $\mathrm{n}^{\circ} 8.080 / 90$ (BRASIL, 1990), na qual o conceito ganha mais amplitude. Para esta Lei, a integralidade é a possibilidade de acesso a todos os níveis do sistema, caso o usuário necessite, como também a possibilidade de integrar ações preventivas com as curativas, no dia a dia dos cuidados realizados nos serviços de saúde. Outros conceitos mais elaborados dilatam o conceito de integralidade, orientando-os para uma prática mais concreta, com a intenção de dar-lhe materialidade na vida dos serviços. Para a Política Nacional da Atenção Básica (PNAB) (BRASIL, 2011), a integralidade é também a integração entre demanda espontânea e demanda programada no fazer dos serviços de saúde, como também é a mescla gerada pelas atividades clínicas e de campo na APS. Essas ditas 'formas 
operacionais' da integralidade garantem a prática da saúde consoante com o que rege a Constituição Federal e 'traduzem', para os profissionais mais 'desavisados', como operar suas práticas sanitárias de acordo com a filosofia do sistema. Contudo, é importante ressaltar que a integralidade como esse esforço para uma oferta que atenda demandas e necessidades construídas é sempre relacional ao contexto.

Ainda sobre a égide dessa discussão, pode-se dizer que a integralidade é um princípio-diretriz do SUS. Princípio porque se vale dessa exegese mais filosófica do ser humano, e diretriz por ter se tornado algo concreto, que guia o processo de trabalho. A integralidade é uma forma de ampliar o olhar dos profissionais para além da lógica da 'intervenção pura', tentando alcançar os contornos do que se compreende como 'cuidar', no âmbito da construção dos serviços de saúde. Na atenção primária, tal como foi concebida sócio-historicamente, o conceito de integralidade subjaz ao discurso operacional (STARFIELD, 2002), contudo, no Brasil, a integralidade é estruturante do SUS, mesmo admitindo-se que o lócus privilegiado para o exercício da integralidade seja a atenção primária. Vale a pena ressaltar como isto se deu.

\section{Conceito de Atenção Primária à Saúde: seletiva ou integral?}

A atenção primária no Brasil, após 2011 (BRASIL, 2011), chancelou-se com o 'rótulo' de Atenção Básica à Saúde (ABS). Exercida em unidades básicas de saúde, por equipes multiprofissionais compostas por médico, enfermeiro, cirurgião-dentista, técnicos de enfermagem, técnicos e auxiliares de saúde bucal, e um conjunto de agentes comunitários de saúde, a multi/interprofissionalidade existente nesses serviços já é um intento de reorganizar o processo de trabalho à lógica de um 'cuidado integral'. A atenção básica, no Brasil, adotou as atividades de educação, vacinação, alimentação e nutrição, saneamento e organização sociocomunitária descritas pela Declaração de Alma-Ata, em 1978 (BRASIL, 2002), com base no conceito de 'cuidados primários em saúde' descrito por tal documento, buscando uma ampliação no escopo das atividades originalmente propostas na citada declaração, em uma tentativa de adaptar o conceito às demandas do País. Assim, foram incorporados ao rol de atividades executadas na atenção básica brasileira procedimentos odontológicos, cuidados específicos em enfermagem e atenção integral a todos os ciclos de vida, transcendendo a tradicional focalização no cuidado materno-infantil. Atualmente, em sua última revisão do escopo de atividades prestadas na atenção básica, a Portaria no 154/2008 (BRASIL, 2008) criou os Núcleos de Apoio à Saúde da Família (Nasf), como forma de expandir o conceito de integralidade no cuidado, através de equipes multiprofissionais (psiquiatra, homeopata, pediatra, fisioterapeuta, fonoaudiólogo, farmacêutico, educadores físicos, nutricionistas, terapeutas ocupacionais e sanitaristas, entre outros). Através de uma lógica de trabalho matricial, as equipes devem ser da modalidade e ter o perfil profissional de acordo com as necessidades epidemiológicas. Estas equipes têm como objetivo garantir o apoio clínico, pedagógico e consultivo às equipes de saúde da família cujos problemas identificados extrapolem suas habilidades/competências específicas.

Mesmo com todos esses elementos, Giovanella (2008) aponta que há limites para o cuidado integral. A autora afirma que o modelo de proteção social, somado aos elementos que constrangem a universalidade (compreendida como acesso universal aos serviços, em contraponto à focalização - ou seja, serviços apenas para a parcela mais pobre da população) tem comprometido a ideia de integralidade em suas múltiplas significações, conforme exposto na seção 
anterior. Isto se agudiza nos períodos de ajustes fiscais dos Estados e promove sérios impactos na prestação dos serviços de atenção básica, que vão desde a precarização dos vínculos de trabalho e cortes de pessoal da saúde, que impactam na composição das equipes, até a redução de suas atividades à forma médico-centrada, desconstruindo, assim, os contornos ampliados da ideia de integralidade, da qual o Brasil é reconhecidamente portador, em âmbito internacional, com uma atenção primária chamada de 'comprehensive care'.

\section{Operacionalização da integralidade no cotidiano das práticas em APS e a formação em saúde}

Para os profissionais que trabalham na atenção básica, a prática do cuidado integral ganha materialidade, tanto em âmbito individual (em consultas compartilhadas e procedimentos humanizados, entre outros, que serão discutidos adiante) como nas atividades que extrapolam os muros das Unidades Básicas de Saúde (UBS) e que recorrem à intersetorialidade como forma de buscar os sujeitos nas suas experiências de vida cotidiana no território, nas quais a cartografia das atividades dos sujeitos ajuda à equipe na compreensão do processo micropolítico em que o cuidado se dá, em ato (MERHY, 1997). Vários elementos, no território, apresentam-se institucionalizados em políticas públicas, e são espaços nos quais a equipe pratica a integralidade em seu conceito mais ampliado. Assim, o Programa Saúde na Escola (PSE), as Academias da Saúde e a Atenção Domiciliar (BRASIL, 2011) são exemplos concretos da interpenetração de políticas públicas no âmbito local, que potencializam a efetividade do direito à saúde e corroboram para que a equipe se mantenha no exercício da prática do cuidado integral, mesmo que suas formações de origem tenham negligenciado essa abordagem em suas graduações (CARVALHO; CECCIM, 2006).

Nesse ponto, é fundamental explicitar que a formação em saúde para atenção primária, mesmo com todos os avanços obtidos, ainda é caudatária da prática integral no exercício das habilidades comuns e específicas dos profissionais de saúde. Em que pese a influência do desenho institucional do sistema de saúde, e suas políticas, na tentativa de alcance do cuidado integral, as universidades e seus docentes ainda padecem de práticas de ensino-aprendizagem que seguem o receituário flexneriano clássico (fragmentadas, especializadas, biologizantes e focadas no modelo liberal). $\mathrm{O}$ papel da universidade $\mathrm{e}$ de seus docentes na elaboração de currículos integrados (ZILBovICIUS, 2011) e no investimento de esforços que articulem o ensino-serviço-comunidade é essencial para gerar um perfil de egressos que seja mais condizente com o que se preconiza para o trabalho em saúde, na perspectiva da integralidade na atenção básica. Só assim, de maneira sinérgica entre a formação e o cuidado, a integralidade alcançará patamares mais elevados na concretude dos serviços de saúde.

\section{Estratégias de humanização em saúde na atenção básica e a integralidade no ato de cuidar}

Com efeito, as políticas públicas que se realizam no território adstrito às UBS corroboram com grande parte da possibilidade do cuidado integral no âmbito da ABS. Entretanto, há um componente importante e bem mais sensível à relação profissional-usuário que está restrito ao encontro entre estes dois sujeitos. Neste sentido, garantir a integralidade na intersubjetividade criada nos múltiplos encontros que os serviços de saúde proporcionam é um desafio no arranjo 
das práticas organizacionais, nas quais a reinvenção do processo de trabalho deve ser sempre ponto de pauta. Com este objetivo, as estratégias de humanização em saúde têm sido convocadas a tentar dirimir a tecnicalidade ainda constitutiva da produção do cuidado em saúde, e têm como desafio promover integralidade nesse microcosmo. Em tese, a humanização é a valorização da dimensão subjetiva do indivíduo, que deve ser priorizada pelos profissionais de saúde nas suas relações cotidianas.

Deslandes (2006) aponta que, se os profissionais de saúde não estão constantemente atentos à dimensão subjetiva do outro, a tendência é a despersonificação do processo de cuidado, através de dois mecanismos principais: a padronização diagnóstica, em função da nosologia patológica, e em detrimento das manifestações singulares dos sinais e sintomas, além de suas especificidades; e o uso intensivo de tecnologias duras, substituindo as interações humanas no encontro com o sujeito.

Assim sendo, diversas estratégias de humanização tentam solucionar essa questão e proporcionar um atendimento integral, que considere o sujeito em sua singularidade. Entre as que mais se aproximam deste objetivo, encontram-se a 'clínica ampliada' e o 'acolhimento'. A clínica ampliada (CAMPOS; AMARAL, 2007) tem como objetivo ser uma clínica do sujeito. Logo, a ideia é considerá-lo, o máximo possível, na sua dimensão biopsicossocial, identificando as correlações entre seus modos de produção de vida e trabalho, em relação ao processo saúde-doença em curso. Em outras palavras, a clínica ampliada tematiza a expressão única da doença no corpo biológico do sujeito, identificando como os sinais e sintomas desse sujeito - que se concretizam de forma difusa e muitas vezes são minorados ou confundem-se com comorbidades - sinalizam a expressividade única da doença nele mesmo. Nesta medida, pode-se dizer, então, que a clínica ampliada é uma não padronização diagnóstica, que se contrapõem àquela própria do ensino biomédico, podendo-se, também, afirmar que esta clínica pretende considerar o sujeito em si, ou seja, é um constante neoartesanato, que, de tanto se realizar, amplia-se em sua capacidade de considerar o humano como um 'holos'.

Outra estratégia que corrobora a prática integral é o acolhimento. Este pode ser entendido como agasalhamento, recebimento, acomodamento, acalento. Ou seja, promove um encontro diferenciado, permeado de contato e afeto. Assim, alguns autores (SANTOS ET AL., 2007) dizem que o acolhimento é uma 'tecnologia do encontro', baseada na escuta qualificada, e que tem como objetivo garantir o acesso do usuário ao serviço, com presteza e corresponsabilização, mesmo quando ele é referenciado para serviços de retaguarda. Neste sentido, pode-se identificar claramente a articulação entre o acolhimento e a integralidade, no conceito de linhas de produção de cuidado, nas quais a integralidade do cuidado (compreendida como acesso do usuário a todos os níveis do sistema) perpassa a rede de atenção, através da qual o usuário - portando seu Projeto Terapêutico Singular (PTS) - é guiado (conceito de usuário-guia), no itinerário terapêutico, pelos serviços, e cujo acesso (enquanto domínio ampliado - desde a identificação da necessidade de saúde até a continuidade do cuidado) requer a acolhida desse usuário, por onde quer que ele passe.

\section{Cuidado, integralidade e Atenção Primária à Saúde: de que articulação estamos falando?}

Quando se fala da articulação conceitual entre cuidado-integralidade-APS, pensa-se em como superar um modelo de atenção tradicionalmente fragmentado, com serviços de saúde atomizados; e qual é o papel da atenção primária nesse cenário. Para a garantia da integralidade em sua multidimensionalidade, é 
necessário que os cuidados com a formação em saúde privilegiem o sujeito como objeto e tragam ao proscênio o cuidado como prática comum às profissões da saúde. A articulação desse modo de produção (AROUCA, 2007) com os serviços de saúde é essencial, e dela depende a capacidade de execução de práticas que transformem a vida e a saúde das populações de territórios adstritos vivos, para concretizar aquilo que a Reforma Sanitária Brasileira (RSB) vislumbrou como 'mudança pela via institucional' (TESTA, 1992). Nesta, o papel dos profissionais de saúde na transformação do modo de produção em saúde, além de perseguir os objetivos técnicos (cuidar da saúde), está alinhado a objetivos políticos bem definidos, tais como executar a reforma 'por dentro' e garantir a passagem para o socialismo, haja vista que o arcabouço constitucional do SUS nada mais é do que um texto de lei que tensiona os princípios filosóficos socialistas art. 196 - com negociações do tipo 'escolha racional', junto ao segmento empresarial dos grandes hospitais privados - art. 199.

Assim, pode-se dizer que o trabalho com a integralidade no cuidado cotidiano do SUS ultrapassa a mera formalidade institucional e está atrelado a um grande projeto societário. É importante ressaltar que, mesmo sob a especificidade do cenário brasileiro, que conformou à articulação cuidado-integralidade-APS essa importância capital no SUS, tal articulação não é privilégio apenas do SUS. Outros sistemas de saúde no mundo (CONILL, 2006) preconizam a integralidade como forma de operacionalização das ações, como nos exemplos conhecidos do Canadá, de Cuba e da Inglaterra. Ainda assim, mesmo com suas atenções primárias cheias de especificidades, a integralidade aparece como constitutiva do cuidado, tanto nas ações individuais quanto nas coletivas. Isto serve de inspiração e desafio para que, no Brasil, persiga-se cada vez mais a qualidade da integralidade das ações de saúde, levando-se sempre em conta os limites e as possibilidades do País.

\section{Limites e possibilidades do cuidado integral na APS}

Como a essência do conceito de integralidade é a ideia de totalidade, imagina-se que seus limites e possibilidades também transitem desde a esfera micro à macroestrutural. Dentre os principais limites para o exercício da integralidade no SUS e, especificamente, na atenção primária, pode-se dizer que a precária interação e o despreparo para lidar com a dimensão subjetiva do ser humano, a fragmentação do processo de trabalho, a constante incorporação e dependência tecnológica da prática em saúde e a formação cartesiana dos profissionais corroboram para práticas de saúde parcelares e isolacionistas, cujas consequências refletem-se em subdiagnósticos, sobremedicações, polifarmácia, sub-registros, sobrerreferenciamentos e tantos outros problemas que decorrem de práticas de cuidado que não têm a integralidade como norte. No âmbito da gestão do sistema, pode-se afirmar que a burocratização e a verticalização, o baixo investimento na qualificação e educação dos trabalhadores, os parcos dispositivos de fomento à cogestão, os desrespeitos constantes aos direitos dos usuários do SUS e a desvalorização dos trabalhadores da saúde são causas que geram diversos efeitos negativos na produção do cuidado em larga escala. Entre os principais efeitos, estão o desestímulo e a apatia para com o processo de trabalho, e a consequente naturalização das condições insalubres; a baixa capacidade de retenção profissional, pois a alta rotatividade gera problemas na criação de vínculos com a população e a incapacidade de vislumbrar o cuidado de forma longitudinal; não reconhecimento do outro como ser autônomo e a manutenção da prática biomédica prescritiva e punitiva, desconsiderando o sujeito em sua singularidade; os atendimentos desumanizados, com períodos de consultas mais curtos e alinhados à lógica da produtividade procedimental etc. 
Entre as principais possibilidades, estudos apontam (SANTOS ET AL., 2007) que, mesmo sob condições adversas, há experiências exitosas no alcance da integralidade. Sanchéz et al. (2010) descreveram que, no cotidiano das práticas dos serviços de saúde, por exemplo, a integralidade tem sido alcançada quando equipes utilizam os diagnósticos sociossanitários para terem visões mais ampliadas do processo saúde-doença no território, o que ajuda a embasar melhor o planejamento de suas ações com vistas à intersetorialidade. Além disto, a presença de estudantes de graduação em unidades de saúde da família tem forçado os profissionais a preocuparem-se com a interprofissionalidade e a sua atuação no território de forma mais frequente. A relação com os usuários tende a ser mais holística em territórios nos quais os profissionais têm vínculos empregatícios mais estáveis e com direitos trabalhistas assegurados, ou ainda naqueles locais/gestões onde o profissional tem capacidade de desenvolver-se em suas competências técnicas ou em relação a projetos de alcance comunitário.

\section{Considerações finais}

Diante do exposto, foi possível perceber a essencialidade do cuidado, da integralidade e da atenção primária no SUS; sua articulação para um processo de produção da saúde que resgate o ser humano como foco das práticas em saúde. Mesmo sob o advento da conjuntura político-social adversa na qual o Brasil atualmente se encontra, é necessário não deixar o debate à míngua e insistir na defesa de um modo de produzir saúde que não esteja às expensas da lógica capitalista, que desumaniza e impede a concretização da saúde como processo civilizatório.

Mesmo sob os auspícios da derrocada do direito à saúde no Brasil, resistir é hoje (re) existir. É reinventar-se e projetar-se para os anos vindouros. A qualificação das armas do argumento com as evidências aqui trazidas faz a defesa do cuidado integral fundante para todos os cidadãos que desejam um futuro melhor para si e suas próximas gerações. Somente assim, é possível avançar na conquista da cidadania. 


\section{Referências}

ADORNO, T. W. Notas de literatura I. Rio de Janeiro: $34,2003$.

AROUCA, S. O dilema preventivista: contribuição para compreensão e crítica da medicina preventiva. São Paulo: Unesp, 2007.

BRASIL. Constituição da República Federativa do Brasil, Diário Oficial [da] República Federativa do Brasil, Brasília, DF, 1988. Disponível em: <www.planalto.gov. br/ccivil_03/constituicao /constituicao.htm>. Acesso em: 22 abr. 2017.

. Lei 8.080, de 11 de setembro de 1990. Diário Oficial [da] República Federativa do Brasil, Poder Legislativo, Brasília, DF, 12 set. 1990.

. Ministério da Saúde. Portaria no 154, de 24 de janeiro de 2008. Cria os Núcleos de Apoio à Saúde da Família (NASF). Diário Oficial [da] República Federativa do Brasil, Poder Legislativo, Brasília, DF, 2008.

Ministério da Saúde. Portaria n ${ }^{\circ} 2.488$, de 21 de outubro de 2011. Aprova a Política Nacional de Atenção Básica, estabelecendo a revisão de diretrizes e normas para a organização da Atenção Básica, para Estratégia de Saúde da Família (ESF) e o Programa de Agentes Comunitários de Saúde (PACS). Diário Oficial [da] República Federativa do Brasil, Brasília, DF, 2011.

. Ministério da Saúde. Secretaria de Políticas de Saúde. Projeto Promoção da Saúde. As Cartas da Promoção da Saúde. Brasília, DF: Ministério da Saúde, 2002.

BUB, M. B. C. et al. Noção de cuidado de si mesmo e conceito de autocuidado na enfermagem. Texto e Contexto em Enfermagem, Florianópolis, v. 15, n. esp., p. 152-157, 2006.

CAMPOS, G. W. S.; AMARAL, M. A. A clínica ampliada e compartilhada, a gestão democrática e redes de atenção como referenciais teórico-operacionais para a reforma do hospital. Ciência \& Saúde Coletiva, Rio de Janeiro, v. 12, n. 4, p. 849-859, 2007.
CARNUT, L. et al. Racionalização das ações intervencionistas e medicamentosas desnecessárias: um ensaio teórico-conceitual. In: CONGRESSO ONLINE DE GESTÃO, EDUCAÇÃO E PROMOÇÃO DA SAÚDE, 5, 2016, São Paulo. Anais... São Paulo: [S. n.], 2016. p. 1-9.

CARVALHO, Y. M.; CECCIM, R. Formação e educação em saúde: aprendizados com a saúde coletiva. In: CAMPOS, G. W. S. et al. (Org.). Tratado de Saúde Coletiva. São Paulo: Hucitec; Rio de Janeiro: Fiocruz, 2006, p. 137-170.

CONILL, E. M. Sistemas comparados de saúde. In: CAMPOS, G. W. S. et al. (Org.). Tratado de saúde coletiva. São Paulo: Hucitec; Rio de Janeiro: Fiocruz, 2006, p. $563-613$.

DESLANDES, S. Humanização: revistando o conceito a partir das contribuições da sociologia médica. In: . Humanização dos cuidados em saúde: conceitos, dilemas e práticas. Rio de Janeiro: Fiocruz, 2006, p. 33-48.

FOUCAULT, M. Resumo do curso. In: EVALD, F. et al. (Org.). A hermenêutica do sujeito. 2. ed., São Paulo: Martins Fontes, 2006.

GIOVANELLA, L. Atenção primária seletiva ou abrangente. Cadernos de Saúde Pública, Rio de Janeiro, v. 24, sup. 1, p. S7-S27, 2008.

MEHRY, E. E. Em busca do tempo perdido: a micropolítica do trabalho vivo em saúde. In: MERHY, E. E.; ONOCKO, R. (Org.). Agir em Saúde: um desafio para o público. São Paulo: Hucitec, 1997.

MENEGHETTI, F. K. O que é um ensaio-teórico. Revista de Administração Contemporânea, Rio de Janeiro, v. 15, n. 2, p. 320-332, 2011.

PINHEIRO, M. R.; MATTOS, R. Construção da Integralidade: cotidiano, saberes, práticas em saúde. 3. ed. Rio de Janeiro: Abrasco, 2004. 
SANCHÉZ, H. F. et al. Integralidade no cotidiano da atenção à saúde bucal: revisão de literatura. Trabalho, Educação e Saúde, Rio de Janeiro, v. 13, n. 1, p. 201-214, 2015.

SANTANA, F. et al. Integralidade do cuidado: concepção e práticas de docentes de graduação em enfermagem no estado de Goiás. Ciência \&t Saúde Coletiva, Rio de Janeiro, v. 15, n. 3, p. 1652-1664, 2010.

SANTOS, A. M. et al. Linhas de tensões no processo de acolhimento das equipes de saúde bucal do Programa Saúde da Família: o caso de Alagoinhas, Bahia, Brasil. Cadernos de Saúde Pública, Rio de Janeiro, v. 23, n. 1, p. 75-85, 2007

STARFIELD, B. Atenção primária: equilíbrio entre necessidades de saúde, serviços e tecnologia. Brasília, DF: Ministério da Saúde, 2002.
TESTA, M. Pensar em saúde. Porto Alegre: Artes Médicas, 1992.

VALE, E. G.; PAGLUICA, L. M. F. Construção de um conceito de cuidado em enfermagem: contribuição para o ensino de graduação. Revista Brasileira de Enfermagem, Brasília, DF, v. 64, n. 1, p. 106-113, jan./fev. 2011.

ZILBOVICIUS, C. et al. A paradigm shift in predoctoral dental curricula in Brazil: evaluating process of changing. Journal of Dental Education, Washington, v. 75, n. 4, p. 557-564, 2011.

Recebido para publicação em junho de 2017

Versão final em setembro de 2017

Conflito de interesses: inexistente

Suporte financeiro: não houve 